\section{(2) OPEN ACCESS}

\title{
Normal human enthesis harbours conventional CD4+ and CD8+ T cells with regulatory features and inducible IL-17A and TNF expression
}

\author{
Abdulla Watad (D) , 1,2,3,4 Hannah Rowe (D) , ${ }^{1}$ Tobias Russell, ${ }^{1}$ Qiao Zhou, ${ }^{1,5}$ \\ Lisa K Anderson, ${ }^{6}$ Almas Khan, ${ }^{7}$ Robert Dunsmuir, ${ }^{7}$ Peter Loughenbury, ${ }^{7}$ Vishal Borse, ${ }^{7}$ \\ Abhay Rao, ${ }^{7}$ Peter A Millner, ${ }^{7}$ Nicola Luigi Bragazzi, ${ }^{8,9}$ Howard Amital, ${ }^{2,3,4}$ \\ Richard Cuhtbert, ${ }^{1}$ Miriam Wittmann, ${ }^{1,10}$ Kassem Sharif, ${ }^{1,2,4}$ Tony Kenna, ${ }^{6}$ \\ Matthew A Brown (D) , ${ }^{6,11}$ Darren Newton (D) , ${ }^{12}$ Charlie Bridgewood, ${ }^{1}$ \\ Dennis G McGonagle"
}

Handling editor Josef $S$ Smolen

- Additional material is published online only. To view please visit the journal online (http://dx.doi.org/10.1136/ annrheumdis-2020-217309)

For numbered affiliations see end of article.

Correspondence to Dr Dennis G McGonagle, Leeds Institute of Rheumatic and Musculoskeletal Medicine, University of Leeds, Leeds, United Kingdom;

d.g.mcgonagle@leeds.ac.uk

AW and HR contributed equally. CB and DGM contributed equally.

Received 11 March 2020

Revised 22 April 2020

Accepted 23 April 2020

Published Online First

13 May 2020

\section{Check for updates}

(C) Author(s) (or their employer(s)) 2020. Re-use permitted under CC BY-NC. No commercial re-use. See rights and permissions. Published by BMJ.

To cite: Watad A,

Rowe $\mathrm{H}$, Russell $\mathrm{T}$,

et al. Ann Rheum Dis

2020;79:1044-1054.

\section{ABSTRACT}

Background The human enthesis conventional T cells are poorly characterised.

Objectives To study the biology of the conventional T cells in human enthesis.

Methods CD4+ and CD8 $+T$ cells were investigated in 25 enthesis samples using immunofluorescence, cytometrically, bulk RNAseq and quantitative real-time PCR following anti-CD3/CD28 bead stimulation to determine interleukin (IL)-17A and tumour necrosis factor (TNF) levels. T-cell receptor (TCR) repertoires were characterised and a search for putative T-cell reactivity was carried out using TCR3 database. The impact of pharmacological antagonism with retinoic acid receptorrelated orphan nuclear receptor gamma $t$ inhibitor (RORyti), methotrexate and phosphodiesterase type 4 inhibitor (PDE4i) was investigated.

Results Immunofluorescence and cytometry suggested entheseal resident CD4+ and CD8+ T cells with a resident memory phenotype (CD69+/CD45RA-) and tissue residency gene transcripts (higher NR4A1/AhR and lower KLF2/T-bet transcripts). Both CD4+ and CD8+ T cells showed increased expression of immunomodulatory genes including IL-10 and TGF- $\beta$ compared with peripheral blood T cells with entheseal CD8+ T cells having higher CD103, CD49a and lower SIPR1 transcript that matched CD4+ T cells. Following stimulation, CD4+ T cells produced more TNF than CD8+ T cells and IL-17A was produced exclusively by CD4+ T cells. RNAseq suggested both Cytomegalovirus and influenza A virus entheseal resident T-cell clonotype reactivity. TNF and IL-17A production from CD4+ T cells was effectively inhibited by PDE4i, while ROR $\gamma$ ti only reduced IL-17A secretion.

Conclusions Healthy human entheseal CD4+ and CD8 + T cells exhibit regulatory characteristics and are predicted to exhibit antiviral reactivity with CD8+ T cells expressing higher levels of transcripts suggestive of tissue residency. Inducible IL-17A and TNF production can be robustly inhibited in vitro.

\section{INTRODUCTION}

The enthesis is the connective tissue junction where ligaments and tendons attach to the bone and is a

\section{Key messages}

What is already known about this subject?

- Recent studies have shown that normal human spinal entheses harbour several innate immune cell populations including type 3 innate lymphoid cells, myeloid cells and also $\gamma \delta \mathrm{T}$ cells. However, a description of adaptive immune T cells at the enthesis has not been reported.

What does this study add?

- This study has shown the presence of entheseal resident conventional T cells with inducible production of interleukin-17 and tumour necrosis factor. The entheseal CD8+ T cells in particular had features of tissue resident memory cells and predicted reactivity to common viral antigens.

How might this impact on clinical practice or future developments?

- The presence of conventional CD4+ and CD8+ T cells in the normal enthesis provides the first direct immunological evidence for adaptive immunity at the enthesis. This provides a basis for studies to explore T cells in the pathology of axial inflammation in spondyloarthropathyrelated disorders.

key target tissue for inflammation in spondyloarthritis ( $\mathrm{SpA})$, including psoriatic arthritis (PsA) and ankylosing spondylitis (AS) where inflammation is evident in both the enthesis soft tissue and the adjacent anchoring perientheseal bone (PEB). ${ }^{1}$ These sites are exposed to mechanical stress and are characterised by microdamage that is accompanied by tissue repair responses that in most cases leads to normal healing. ${ }^{2-5}$ However, in genetically prone subjects, those with dysbiosis and other factors, the post-inflammation repair processes may be excessive and eventually lead to new bone formation. ${ }^{367}$

Animal model studies suggest the primacy of enthesis organ disease in experimental SpA which was originally shown in the DBA-1 mouse model 
and also in tumour necrosis factor (TNF) transgenic models and subsequently in other models. ${ }^{89}$ Given the fibrocartilageneous and ligamentous/fibrous nature of the enthesis, the actual insertion region was not thought to contain cells of the immune system. However, several pioneering studies showed the presence of a novel T-cell population in murine interleukin (IL)-23/IL-17 axisdependent disease. ${ }^{8}$ In mouse models, activated V $\gamma 6+\mathrm{CD} 27-\gamma \delta$ $\mathrm{T}$ cells were found in uninflamed normal entheseal tissue and constituted the largest resident T-cell subset. ${ }^{10}{ }^{11}$ Galvanised by these novel animal model experiments, we reported that normal human spinal entheses harbour several innate immune cell populations including type 3 innate lymphoid cells (ILCs), myeloid cells and also $\gamma \delta \mathrm{T}$ cells that express IL-23R, with inducible IL-17A and IL-17F transcript expression. ${ }^{12} 13$

A remarkable feature of human SpA-related disorders are the multiple genetic associations suggesting a role for adaptive immunity, especially conventional $\mathrm{T}$ cells, in disease immunopathogenesis-ranging from MHC class-I associations in AS and PsA to ERAP-1 and RUNX3 SNPs (single nucleotide polymorphisms) among others. ${ }^{14}{ }^{15}$ The most prominent MHC class-I association, HLA-B27, is present in $\sim 90 \%$ of AS patients pointing to CD8 + Tcells in pathogenesis. ${ }^{16}$ Conventional T cells are known to be present in the enthesis along with PEB infiltrating lymphocytes in patients with $\mathrm{SpA} .{ }^{17}$ Indeed, the anchoring PEB is the major site of inflammation or osteitis in AS. The identification of tissue-resident memory T cells (TRM) that occupy non-lymphoid organs such as skin, lung and the gastrointestinal tract raises the possibility that such cells are also resident within normal enthesis. ${ }^{18}$ TRM cells are highly heterogeneous with respect to anatomical positioning, phenotype, molecular regulation and effector function. ${ }^{19}$

A cardinal feature of the enthesitis-associated pathology of SpA is the response to TNF and IL-17A inhibition, ${ }^{20}{ }^{21}$ but the biological basis for this remains undefined given the rudimentary knowledge about human enthesis immunity. However, a recent clinical trial of IL-23 blockade reported disappointing results in AS patients, ${ }^{22}$ suggesting a mechanism for IL-17 production independent of IL-23 at the enthesis. ${ }^{23}$ These translational therapeutic insights suggest that TRM cells might be key IL-17A and TNF producers. In the current study, we aimed to characterise human entheseal CD4+ and CD8 + Tcells and assessed their ability to express SpA-related cytokines including TNF and IL-17A following CD3/CD28 stimulation. We also evaluated the impact of SpA relevant therapeutic agents (methotrexate (MTX) and inhibitors (i) of PDE4 and ROR $\gamma t$ ) on attenuating TNF and IL-17A production towards efforts to develop an in vitro model system for predicting potential drug efficacy for enthesitis.

\section{MATERIALS AND METHODS \\ Patients and samples}

The study protocol was approved by the North West-Greater Manchester West Research Ethics Committee. Human interspinous process and matched peripheral blood were obtained from 25 patients (with informed written consent) (mean age of $42.4 \pm 27$, median age of 39 , and female to male ratio of $3: 2$ ) who underwent elective spinal surgery for either decompression or scoliosis correction using methods previously reported. ${ }^{12}$ Further details regarding the complete patient cohort are reported in online supplementary table 3.

\section{Isolation of primary cells from enthesis and matched blood}

Entheseal samples were separated into entheseal soft tissue (EST) and PEB and both were enzymatically digested as previously described (online supplementary figure 1). ${ }^{12}$ For both cell preparations, blood and entheseal cells, density gradient separation (Lymphoprep) was conducted in order to obtain peripheral blood mononuclear cells (PBMCs) and entheseal mononuclear cells (EMCs), respectively, using methods previously described. ${ }^{24}$

\section{Immunophenotyping of entheseal and peripheral blood derived T cells}

EMCs or PBMCs were stained with zombie aqua (live/dead discrimination), anti-CD45 (to exclude non-leucocytes), CD3 (T-cell inclusion), CD4 and CD8. Tissue resident memory cells were identified by CD69+ and naive/circulating cells by CD45RA+. All flow cytometry was conducted on the Cytoflex LX (Beckman Coulter) and subsequent analysis completed using the CytExpert Acquisition and Analysis Software (V.2.3) and FlowJo software (Tree Star, USA). A full list of antibodies, clones and fluorophores can be found in online supplementary table 1 .

\section{Histology and immunofluorescence microscopy}

For immunofluorescence microscopy, frozen sections of EST were incubated with an Alexa Fluor 647 labelled antibody against CD3 (clone UCHT1, BD Biosciences) and counterstained with 4',6-diamidine-2'-phenylindole dihydrochloride.

\section{Magnetic cell separation}

Following isolation of EMCs from digested entheseal samples and PBMCs from processed blood, CD4+ and CD8 + Tcells were isolated using biotinylated anti-CD4 or CD8 (both from Miltenyi Biotech). Cells were isolated using magnetic separation (Miltenyi Biotech LS columns), according to the manufacturer's instructions.

\section{Cell culture}

Following cell isolation, CD4+ and CD8 + Tcells were plated out in a 96-well plate (minimum of $5 \times 10^{4}$ cells/well) in RPMI (GIBCO) containing $10 \%$ fetal calf serum and $1 \%$ penicillin/ streptomycin. For inhibition studies, cells were incubated with the following compounds for 1 hour in standard culture conditions $\left(5 \% \mathrm{CO}_{2}, 37^{\circ} \mathrm{C}\right)$ : ROR $\gamma$ t inhibitor $10 \mu \mathrm{M}^{25}$ (Pfizer), MTX, $5 \mathrm{mg} / \mathrm{mL}$ (Cayman Chemical), rolipram (a PDE4 inhibitor) $10 \mu \mathrm{M}$ (Cayman Chemical) or dimethyl sulfoxide (DMSO) control (0.1\%). All drugs were diluted to maintain a final concentration of $0.1 \%$ DMSO. Cells were stimulated using anti-CD3/ CD28 (GIBCO) for 48 hours. To test the potential of compounds to affect cell viability, flow cytometry was used to assess CD45+, $\mathrm{CD} 3+, \mathrm{CD} 4 / \mathrm{CD} 8+$ and live dead viability by aqua zombie.

\section{TNF and IL-17A determination by ELISA in entheseal stimulated cell supernatants}

Following 48 hours of stimulation, cells were removed by centrifugation and supernatant was stored at $-80^{\circ} \mathrm{C}$. Concentrations of TNF and IL-17A were measured using sandwich ELISAs from eBioscience/Thermo Fisher (Waltham, Massachusetts, USA). ELISAs were carried out according to the manufacturer's protocol. Following this, $\mathrm{pg} / \mathrm{mL}$ and $\mathrm{pg} /$ cell were calculated.

\section{Intracellular flow cytometry for protein measurement}

Enthesis mononuclear cells, $5 \times 10^{6}$, were plated out and stimulated with anti-CD3/CD28 as before in the presence of Golgi plug (BD) for 3 hours. The cells were fixed using Intraprep kit (Beckman Coulter) and stained intracellularly with IL-17A and TNF. 


\section{Quantitative real-time PCR for transcript analysis}

$\mathrm{T}$ cells were sorted using an Influx (BD) cell sorter directly into RNA extraction buffer, supplied as a component of the PicoPure RNA isolation kit (Thermo Fisher). cDNA was synthesised with a reverse transcription kit (Fluidigm) then underwent preamplification (18 cycles) using a pre-amp master mix (Fluidigm) with a solution containing all primer sets. All primers were purchased from Applied Biosystems. Transcript analysis was performed by qPCR using the Biomark HD gene expression system (Fluidigm); values displayed are $\log 10 \Delta \mathrm{Ct}$ relative to the HPRT1 housekeeping gene.

qRT-PCR was completed using the TaqMan Gene expression assay as per the manufacturer's instructions (Applied Biosystems). Samples, no template control (NTC) and positive controls (pooled cDNA) were loaded onto either a MicroAmp Optical 96-Well Reaction Plate (Applied Biosystems) or a MicroAmp Optical 384-Well Reaction Plate (Applied Biosystems) and run on either the Quantstudio 5 or Quantstudio 7 instrument, respectively (both from Applied Biosystems) using the Quantstudio Design and Analysis software (V. 1.4, Thermo Scientific), the Comparative Threshold $(\mathrm{Ct})$ value was subsequently normalised using the HPRT1 housekeeping gene and unstimulated control for $2^{-\Delta \Delta \mathrm{Ct}}$. All primers were purchased from Applied Biosystems. A full list of primers used can be found in online supplementary table 2 .

\section{RNAseq}

Bulk RNAseq was performed on CD3 $+\mathrm{CD} 45+\mathrm{CD} 4^{+}$or $\mathrm{CD} 8^{+}$ T-cell subsets isolated from entheseal digests $n=4$ for both PEB and EST. RNA was then purified using the PicoPure RNA isolation kit (Thermo Fisher), RIN (RNA Integrity Number) scores and concentration in $\mathrm{ng} / \mu \mathrm{L}$ were determined by Tape Station prior to RNAseq analysis. Following this, MiXCR (V.3) was used to extract clonal TCR rearrangements. CDR3 (complementary determining region 3 ) amino acid sequences were used to interrogate the TCR3 database (https://tcr3d. ibbr.umd.edu/) in order to assess epitope specificities based on model-based scoring matrices (PAM-30). Further details of bulk RNAseq analysis using the Galaxy platform are displayed in online supplementary methods 1 .

\section{Statistical analysis}

Analysis was performed using GraphPad Prism software (GraphPad Software, La Jolla, California, USA) and statistical analysis was performed in SPSS (IBM SPSS Statistics V.25, USA). Error bars represent the SE of the mean (SEM); refer to the Results section for specific tests used per experimental procedure.

\section{RESULTS}

\section{Conventional CD4+ and CD8+ T cells are present in the human enthesis}

To investigate entheseal T-cell topography, anti-CD3 immunofluorescence staining was used (tonsil tissue used as a positive control) (figure 1A-C). EST T cells were sparse and typically located in small clusters (figure 1C). Following enzymatic digestion of the samples, flow cytometry was used identify CD4+ and CD8 + Tcells from the enthesis (figure 1D). Clear populations of $\mathrm{CD} 4+$ and $\mathrm{CD} 8+\mathrm{T}$ cells were readily identifiable.
Transcriptional and flow cytometric profiling of entheseal CD4+ and CD8+ T cells compared with peripheral blood supports TRM status

Comparison of blood-derived and entheseal-derived (from both PEB and EST) CD4+ and CD8 + Tcell transcripts suggests that entheseal $\mathrm{T}$ cells are tissue resident with higher $A h R$ and NR4A1 transcript expression. Contrastingly, transcripts indicative of circulating T cells, such as KLF2 and T-bet, show higher transcript expression in blood $\mathrm{T}$ cells (figure 2A). Entheseal CD4+ and CD8 + Tcells also show higher expression of growth factors and molecules associated with tissue repair and homeostasis, such as vascular endothelial growth factor A (VEGF-A), transforming growth factor beta 1 (TGF$\beta 1)$ and IL-10 when compared with matched peripheral blood (figure 2A).

Flow cytometry was used to further phenotype both blood and entheseal $\mathrm{T}$ cells. Entheseal-derived CD4+ and CD8 + T cells showed significantly higher CD69 expression, supporting a tissue resident memory (TRM) phenotype (figure 2B). In contrast, a greater percentage of peripheral blood $\mathrm{T}$ cells showed CD45RA expression compared with entheseal-derived $\mathrm{T}$ cells, further supporting tissue residency for entheseal $\mathrm{T}$ cells (figure $2 \mathrm{~B}$ ).

\section{Induction of IL-17A and TNF in entheseal CD4+ and CD8+ T cells}

Following anti-CD3/CD28 stimulation, a significantly greater proportion of entheseal-derived $\mathrm{CD} 4+\mathrm{T}$ cells increased IL-17A and TNF production when compared with unstimulated. There was a significant increase in the proportion of $\mathrm{CD} 8+$ enthesis $\mathrm{T}$ cells upregulating TNF production, and although IL-17 production trended upwards following stimulation, this was not statistically significant (figure 3).

\section{Assessment of entheseal bone and soft tissue CD4+ and CD8+ T-cell repertoire}

Following RNASeq, the initial analysis of conventional $\alpha \beta$ TCRs highlighted the marked reduction in clonal diversity in EST compared with PEB conventional T cells, where there was evidence to suggest that PEB subpopulations are more diverse than their EST counterparts. However, more sorted cells were generally present in PEB compared with EST, where CD4+ populations contained (17 $353 \pm 6147$ cells and $1419 \pm 988$ cells, respectively) (figure $4 \mathrm{~A}$ ), the same trend is also seen in CD $8+$ populations (20 524 \pm 5545 cells and $1384 \pm 1076$ cells, respectively) (figure 4B). We assessed T-cell clones for potential viral reactivity, by looking at epitope specificities from the TCR3 database (https://tcr3d.ibbr.umd.edu/); out of the 13 potentially reactive clonal sequences, the most common viral targets were Cytomegalovirus (CMV) in eight sequences and influenza A virus (IAV) in two sequences.

Bulk RNAseq analysis of the CD4+ and CD $8+T$ cells $(n=6$ in total) was undertaken for tissue residency markers including CD69, CD103, CD49a and S1PR1 following normalisation with the GADPH housekeeping gene. As confirmed by flow cytometry CD69 transcript was substantially higher in enthesis compared with blood but the other transcripts were comparable between enthesis and blood. However, in four entheseal samples, CD103 and CD49a transcripts were 3.8-fold and 4.6-fold higher in entheseal CD8 + Tcells, respectively, compared with CD4+ entheseal T cells with S1PR1 transcripts being correspondingly reduced. From other studies, it appears 

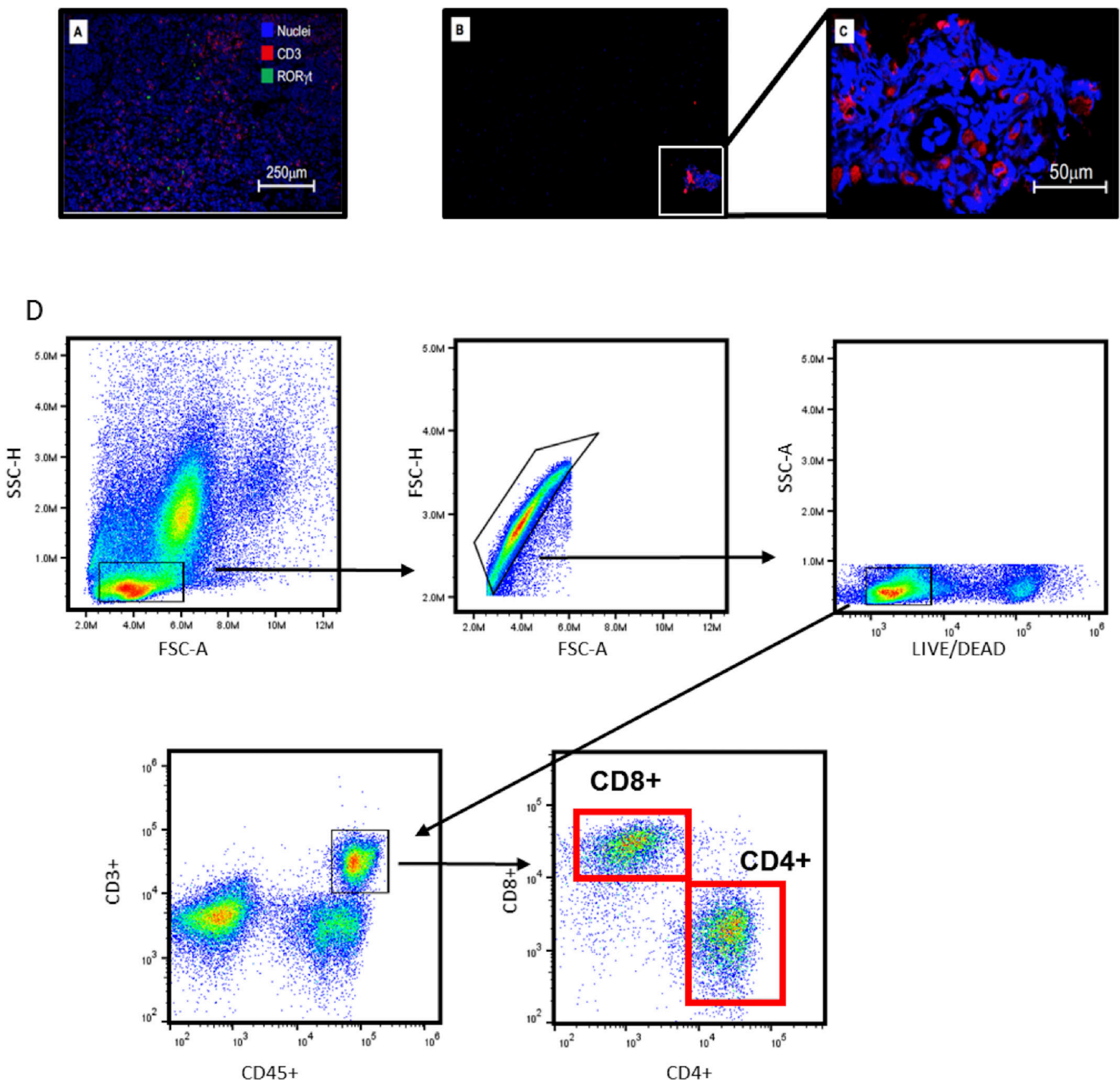

Figure 1 Immunofluorescence and cytometry for entheseal CD4+ and CD8+ Tcells. Anti-CD3 immunofluorescence staining showing T cells in tonsil as positive control (A), in enthesis at lower power (B) and at higher power (C) where a small region of CD3 positivity in (B) is magnified. (D) Phenotypic identification of CD4+ and CD8+ T cells by fluorescence-activated cell sorting (FACS) following digestion of human enthesis. Following doublet exclusions, cells were gated on CD45+ (all white cell count) and CD3+ (all T cells); this gate was then further subdivided into CD4+ and CD8+ Tcells.

that expression of CD69 leads to the inactivation and internalisation of S1PR1 which supports our concept of these cells showing tissue residency. ${ }^{26}$

\section{Analysis of regulatory markers in entheseal and blood-} derived T cells

Given the high basal TGF $\beta$ transcript expression and the absence of proinflammatory cytokine transcripts (figure 2A), we further investigated whether entheseal $\mathrm{T}$ cells were regulatory in nature. While a population of Tregs $(\mathrm{CD} 4+\mathrm{CD} 25+$ FoxP3+) was readily identifiable in the blood, this was not the case in the enthesis (figure $5 \mathrm{~A}$ ). To further induce a regulatory phenotype, we used a series of clinically relevant compounds to assess their effects on T-cell regulatory phenotype markers (FoxP3 and TGF31). Isolated T cells were stimulated with anti-CD3/CD28 with and without ROR $\gamma$ t inhibitor, MTX and PDE4 inhibitors (figure 5B-D). Only the phosphodiesterase type 4 inhibitor (PDE4i) significantly upregulated FoxP3 expression in entheseal CD4+ T cells (figure $5 \mathrm{~B}$ ). With regard to the expression of TGF $\beta$, the therapeutic agents resulted in no significant changes in expression, except for entheseal CD4+ Tcells, where PDE4itreated cells showed higher expression (figure 5C). MTX significantly reduced the expression of FoxP3 from entheseal CD8+ Tcells (figure 5D). 
A

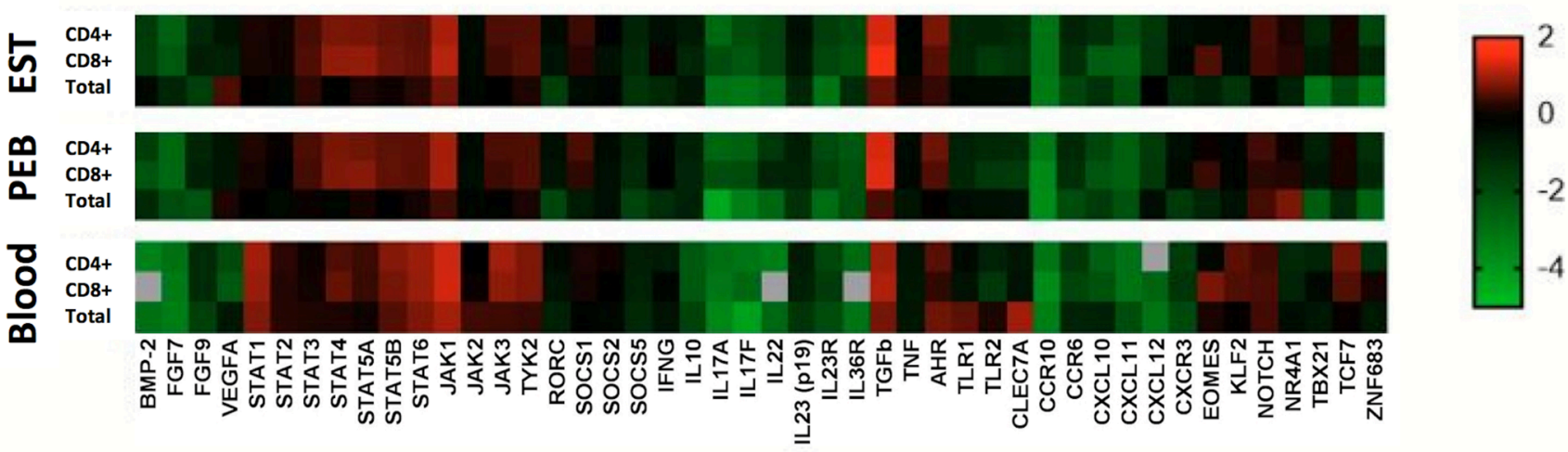

B

CD4+ circulating memory

CD4+ resident memory

CD8+ circulating memory

CD8+ resident memory
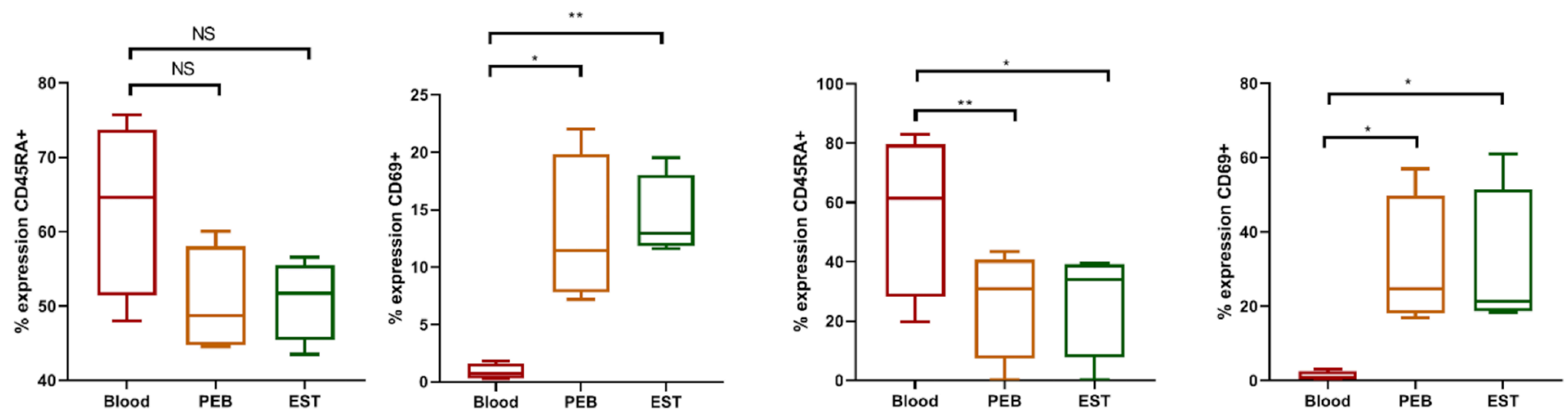

Figure 2 Transcriptional and flow cytometric profiling of entheseal T cells. CD4+ and CD8+ T cells were sorted from EST, PEB and peripheral blood (PB). Basal expression of cytokines, chemokines, growth factors, signalling molecules, tissue residency markers was assessed (A). Colour coding refers to differentially expressed genes where values less than -1 indicate low expression and values greater than 1 indicate higher expression, those with grey boxes indicate no values, values displayed are log $10 \triangle \mathrm{Ct}$ relative to HPRT ( $\mathrm{n}=16$ samples). Flow cytometry (B) was used to assess the $\%$ of circulating memory T cells (CD69- CD45RA+) and resident memory T cells (CD69+CD45RA-) in both CD4 +and CD8+ T cells in PEB, EST and PB paired t tests, $n=4 .{ }^{*} p<0.05 ;{ }^{*} p<0.01 ;{ }^{* * *} p<0.001$. EST, entheseal soft tissue; PEB, perientheseal bone.

\section{RORyti, MTX and PDE4i impact on enthesis CD4 and CD8 T-cell cytokine production}

We next evaluated the effect of disease relevant drugs on entheseal and blood-derived $\mathrm{T}$ cells including those not thought to be generally efficacious for enthesitis such as MTX and those that may show efficacy including phosphodiesterase type 4 inhibitor (PDE4i) and retinoic acid receptor-related orphan nuclear receptor gamma $t$ inhibitor (ROR $\gamma t i)$. The latter was evaluated as ROR $\gamma t$ is a key transcription factor driving Th17 cell differentiation, and production of IL-17A, IL-17F and IL-22 in innate and adaptive immune cells. ${ }^{27}$ Compounds were tested for their ability to attenuate TNF and IL-17A secretion. CD4+ and CD8+ T cells were isolated from the enthesis and matched blood, and stimulated with anti-CD3/CD28, and TNF and IL-17A were measured by ELISA. CD4+ T cells robustly secreted IL-17A and TNF, whereas CD8+ only secreted TNF (IL-17A was undetectable, data not shown). Both PDE4i and ROR $\gamma$ ti significantly reduced IL-17A secretion from both blood and enthesis-derived $\mathrm{CD} 4+$ cells (figure 5F), whereas MTX failed to significantly attenuate IL-17A secretion in CD4+ entheseal-derived cells; however, MTX did attenuate IL-17A secretion in blood. TNF derived from both entheseal and blood-derived CD4+ Tcells was significantly attenuated by PDE4i, but not ROR $\gamma$ ti or MTX (figure 5F). Similarly, CD8 + T-cell-derived TNF was attenuated by PDE4i, but not ROR $\gamma$ ti or MTX (figure $5 \mathrm{G}$ ).

Cell viability was tested for all three compounds with none of the compounds significantly altering either entheseal CD4+ or $\mathrm{CD} 8+\mathrm{T}$ cells (figure $5 \mathrm{H}$ ). Next, we assessed any potential difference of drug inhibition between the entheseal and blood-derived T cells (figure 5I). CD4+ IL-17A inhibition by ROR $\gamma$ ti showed no significant difference between enthesis and blood. For both T-cell subsets, there was no significant differences between inhibition of TNF or IL-17A by PDE4i between the enthesis and blood (figure 5I). The findings from peripheral blood-derived $\mathrm{T}$ cells with drug inhibition were generally similar to the enthesis.

\section{DISCUSSION}

We report, for the first time, the presence of conventional CD4 + and CD8 + T cells in the human enthesis including PEB and spinal soft tissue. In health, these exhibited regulatory features including high TGF $\beta 1$ transcripts, but low proinflammatory cytokine transcript levels and protein production. Unlike for matched blood, there was no clear FoxP3 regulatory T-cell population identifiable at the healthy enthesis, although T-cell stimulation in the presence of PDE4 inhibition was 
$\mathbf{A}$

CD4+ TNF

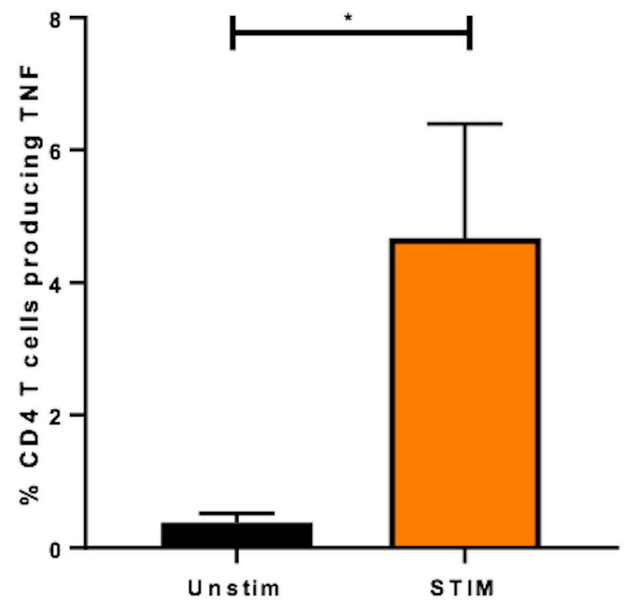

CD4+ IL-17A

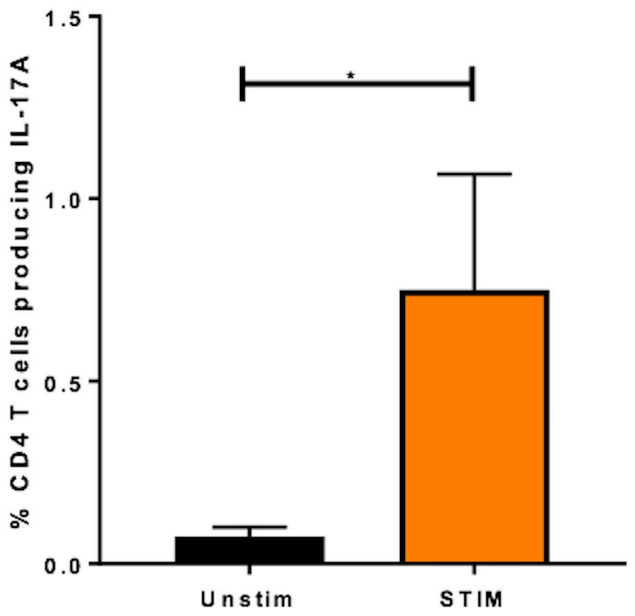

CD8+ TNF

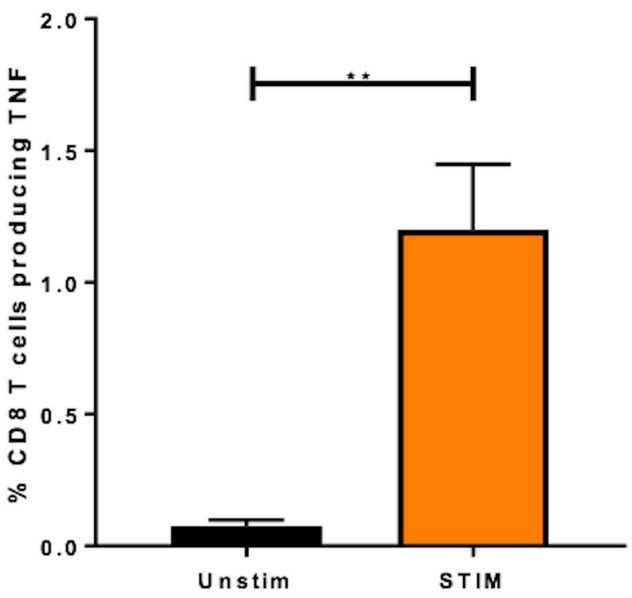

CD8+ IL-17A

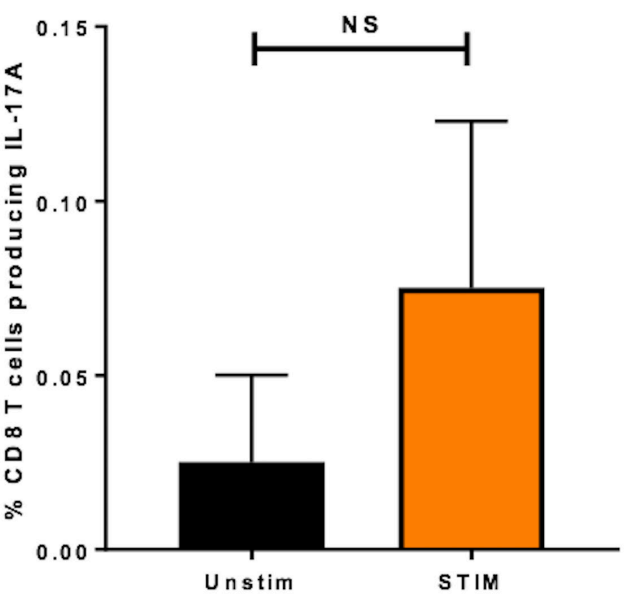

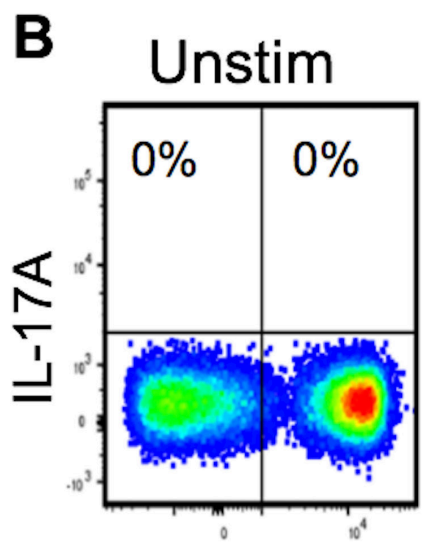

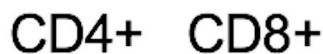

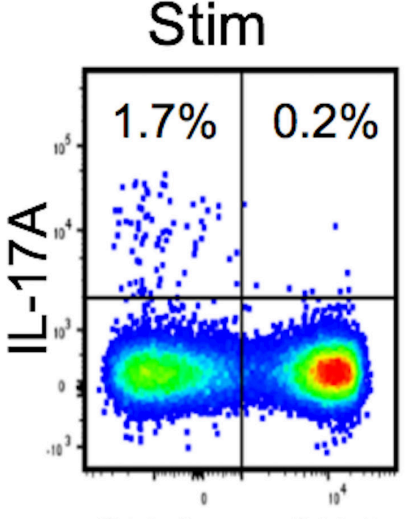

CD4+ CD8+

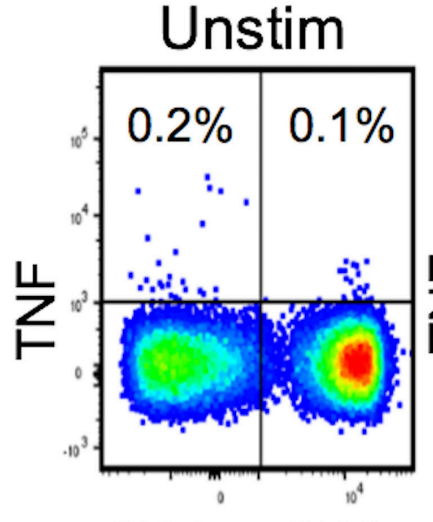

CD4+ CD8+

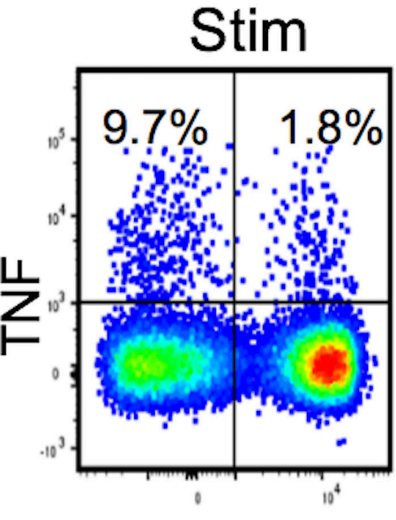

CD4+ CD8+

Figure 3 Intracellular interleukin (IL)-17A and tumour necrosis factor (TNF) induction in entheseal CD4+ and CD8+ T cells. Following digestion of human entheseal samples (PEB), cells were stimulated with anti-CD3/CD28 in the presence of Golgi plug for 3 hours. CD4+ and CD8+ T cells were subsequently stained intracellularly for TNF and IL-17A. TNF or IL-17A was quantified (A) following assessment by flow cytometry (B). $n=3$. Paired $t$ tests. ${ }^{*} p<0.05 ;{ }^{* *} p<0.01 ;{ }^{* *} p<=0.001$. 
A

CD4+ Total $\alpha \beta$ CDR3 Reads

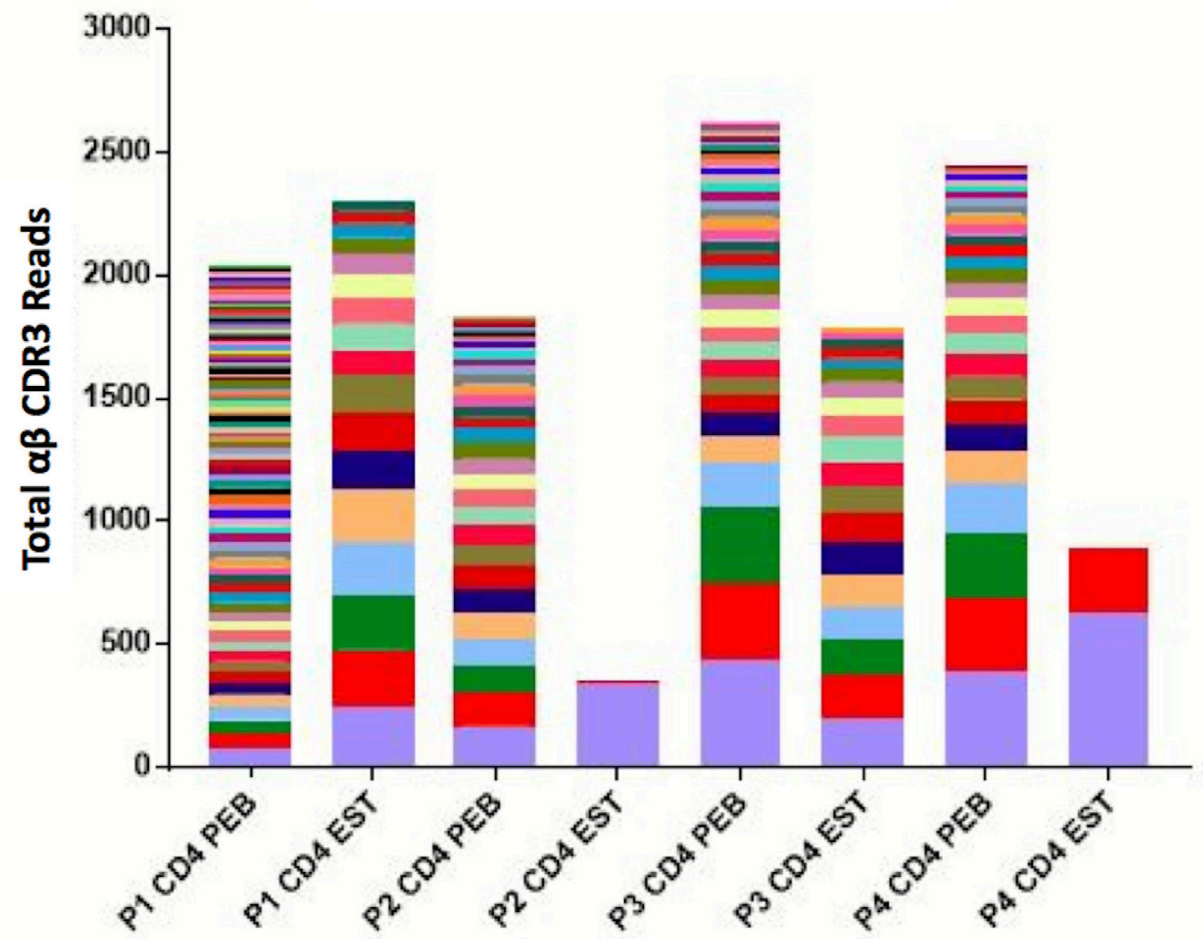

B
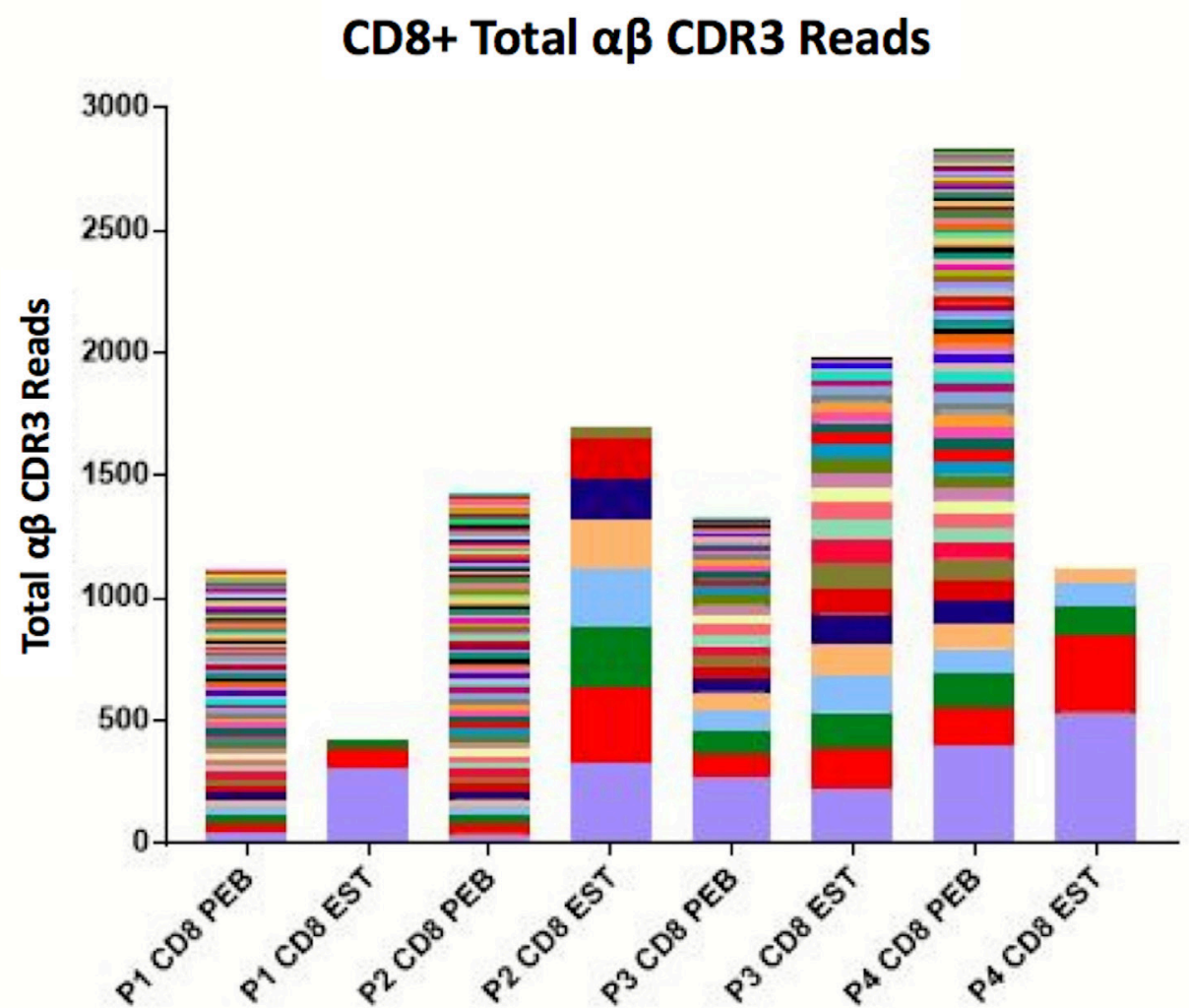

Figure 4 Assessment of the conventional T-cell repertoire in peri-entheseal bone (PEB) and entheseal soft tissue (EST) within the CD4+ and CD8+ T-cell subpopulations. Following MiXCR outputs, RNAseq data were used to determine potential clonality within the T-cell subpopulations, from both PEB and EST in both CD4+ (A) and CD8+ (B) T-cell populations where each individual clone is represented by a different colour. $n=4$ ( 2 female, 2 male); mean age $=30.2$ years. Where $\mathrm{P} 1$ relates to the first patient, $\mathrm{P} 2$ relates to the second patient, $\mathrm{P} 3$ relates to the third patient and $\mathrm{P} 4$ relates to the fourth patient. 


\section{Blood}

\section{Enthesis}

A

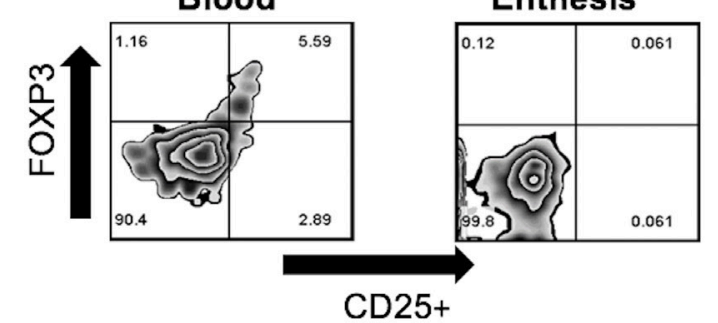

B

CD25+

C

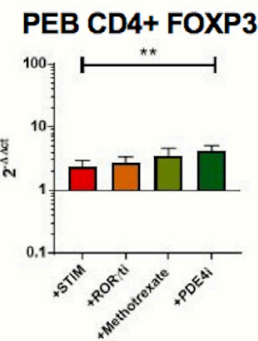

PB CD4+ FOXP3

PEB CD4+ TGF $\beta$

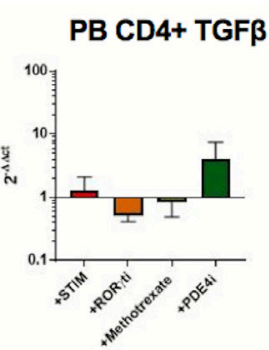

D
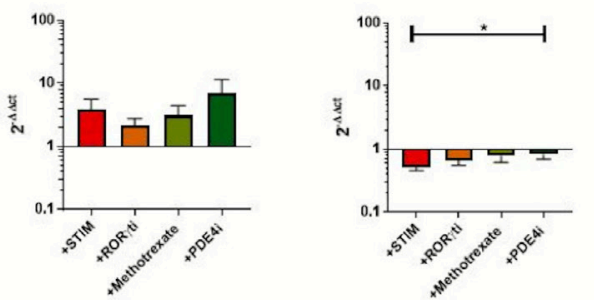

E

PB CD8+ FOXP3

PEB CD8+ TGF $\beta$
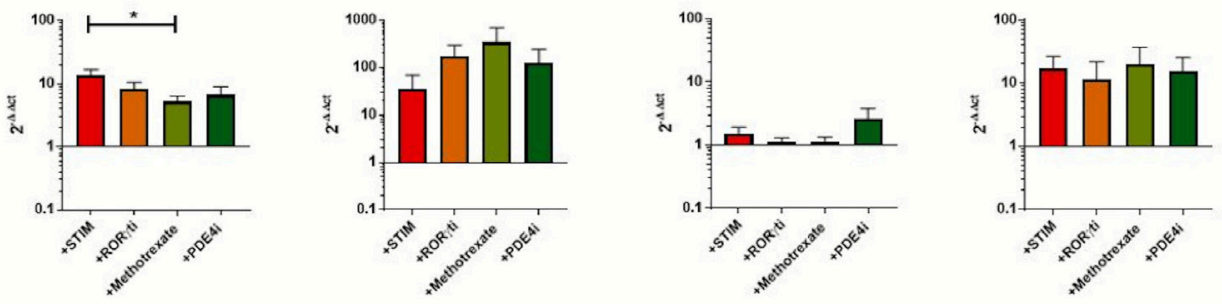

PEB CD4+ IL-17A

PB CD4+ IL-17A

PEB CD4+ TNF

PB CD4+ TNF
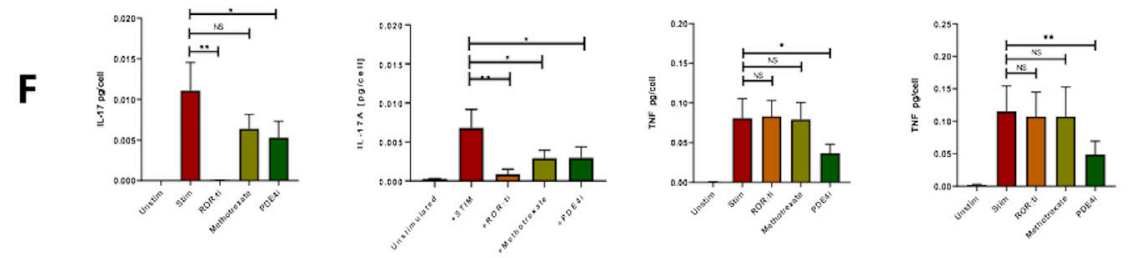

G
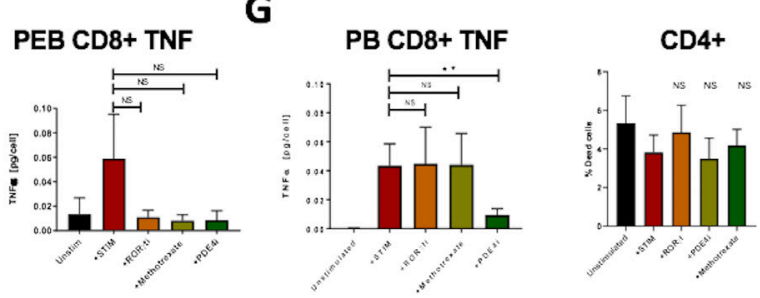

H

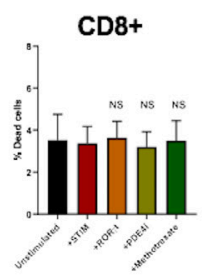

CD4+ TNF PDE4i

CD4+ IL-17 RORyti

CD4+ IL-17 PDE4i

CD8+ TNF PDE4i
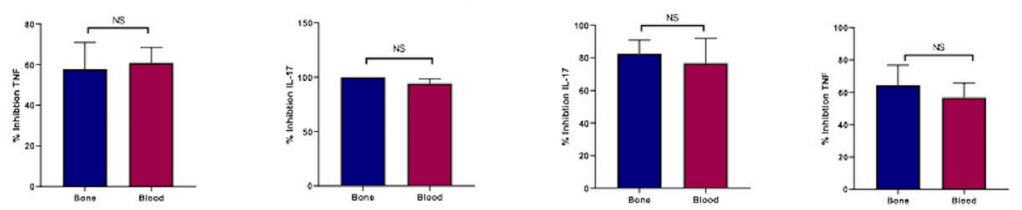

Figure 5 The effect of compounds of regulatory and inflammatory protein and gene expression basal levels of FOXP3 were measured by flow cytometry in unstimulated perientheseal bone (PEB) and peripheral blood mononuclear cell (PBMC) and show a clear population of Tregs in blood but not the enthesis (A). CD4+ and CD8T cells were isolated from PEB or matched peripheral blood and stimulated with anti-CD3/CD28 for 48 hours with and without therapeutic agents indicated. QRT-PCR was used to determine the expression of FOXP3 and TGF $\beta 1$. (B-E) $2^{-\triangle \Delta C t}$ was used to measure relative expression fold change. IL-17A and TNF protein secretion was measured by ELISA (F-G) $(n=8)$. The effect of therapeutic agents on CD4+ and CD8+ viability following a 48-hour incubation $(H), n=3$. The difference in efficacy between phosphodiesterase type 4 inhibitor (PDE4i) and retinoic acid receptor-related orphan nuclear receptor gamma t inhibitor (ROR $\gamma \mathrm{ti}$ ) in attenuating cytokine secretion in enthesis (PEB) compared with blood in CD4+ populations and CD8+ populations (I). One-way t test, ${ }^{*} p<0.05 ;{ }^{* *} p<0.01$. 
capable of FoxP3 transcript upregulation. We also confirmed that these entheseal $\mathrm{T}$ cells have the capability to secrete pivotal disease-relevant cytokines such as TNF and IL-17A following CD3/CD28 stimulation, without the use of other exogenous cytokines such as IL-23. Enthesis-derived CD4+ T cells secreted both TNF and IL-17A following stimulation, whereas enthesis-derived CD8 + T cells only produced clearly detectable TNF.

Through mouse models and clinical observations, both TNF and IL-17A have been heavily implicated in enthesitis-related pathology. ${ }^{1028}$ While innate lymphocytes are major inflammatory cytokine producers in mice, ${ }^{11}$ our findings confirm that conventional T-cell populations that can express these cytokines are present at the normal enthesis. Given the MHC-I and MHC-II associations with human SpA spectrum disorders, our findings highlight the importance of the investigation of entheseal $\mathrm{T}$ cells in pathological conditions such as AS and PsA.

Central to the T-cell antigen response is the CDR3 (Complementary determining region 3 ) which provides both antigen specificity and affinity. The bulk RNAseq data looking for CDR3 showed a more diverse repertoire in PEB than EST, arguably this could be due to the haematopoietic marrow that PEB contains. The ability to demonstrate clonal TCR usage provides a useful tool to dissect the immunopathology of inflammatory diseases, when looking at the epitope specificities from the TCR3 database (https://tcr3d.ibbr.umd. $\mathrm{edu} /$ ), and out of the 13 sequences with potential targets, the most common finding was CMV in eight sequences and IAV in two sequences, respectively. Bulk RNAseq highlights the presence of clonal populations in both CD4+ and CD8+ conventional T-cell populations with the latter scoring higher on model-based scoring matrices (PAM-30). Overall, these results showing high diversity and limited clonality in PEB and vice versa in the EST suggest that the conventional T cells in each of these compartments are distinct. EST T cells show a limited repertoire, while PEB T cells maintain their capacity to respond to a range of diverse antigens. This likely reflects the greater immune cell numbers that accumulate at the PEB, which in addition to being the target site for inflammation in AS also contains normal bone marrow.

The extremely low levels of IL-17A production from entheseal CD8 + T cells in comparison to CD $4+$ T cells from both blood and enthesis following stimulation reinforces the view that CD4+ Tcells are more important for cytokine production than CD8 + T cells. ${ }^{29}$ CD8 + IL-17 producing (Tc17) cells have been identified in PsA synovial fluid, where they exhibit a tissue resident memory (TRM) phenotype, with most present in the effector tissues and a small number continuing to recirculate in the secondary lymphoid organs ready for antigen re-encounter. ${ }^{30}$ Tc17 cells are highly plastic in nature and can switch to interferon- $\gamma$ producing Tc1 cells in certain tissues. ${ }^{31}$

Entheseal CD4+ and CD8 + Tcells are transcriptionally, phenotypically and functionally distinct from matched recirculating peripheral T cells. Entheseal T cells also have higher expression of transcripts involved in repair and tissue homeostasis, such as IL-10, TGF $\beta 1$ and VEGF-A when compared with their blood counterparts. In the large majority of cases, these repair processes consist of self-limited inflammation and may lead to the complete healing of the damaged tissue. ${ }^{32}$ However, in some cases, in those subjects with genetic predisposition, enthesitis may exhibit chronicity as is the case in SpA-associated disorders where it is considered a pathognomonic feature. ${ }^{33}$
The ability of disease relevant compounds to attenuate IL-17A and TNF production was also evaluated. It is well known that ROR $\gamma \mathrm{t}$ is a transcription factor and regarded as the master regulator of IL-17A. ${ }^{34}$ ROR $\gamma t$ antagonists have been investigated in numerous murine models of inflammation and autoimmunity, yielding promising results. ${ }^{35} 36$ In human studies, ROR $\gamma$ t inhibition was recently shown to inhibit IL-17A from synovial T cells from both PsA and AS patients and also blood $\mathrm{T}$ cells. ${ }^{37}{ }^{38}$ We also showed that rolipram, a PDE4 inhibitor, was able attenuate TNF and IL-17A secretion CD4 + T cells and TNF from CD8 + T cells. Our system may provide a model for testing the impact of drugs on the adaptive immune system in preclinical work in SpA but clear difference between activated peripheral blood cells undergoing drug antagonism were not evident.

The $\mathrm{T}$ memory subsets are crucial for maintaining immunological memory and include TCM ( $\mathrm{T}$ central memory CCR7+ and CD45RA-), TEM (T effector memory, CCR7- and CD45RA), TRM (tissue resident memory cells, CD69+CD45RA- and CD103 + in CD8 + subpopulations) and TSCM a subset of TCM cells (T memory stem cells, CCR7+C$\mathrm{D} 62 \mathrm{~L}+\mathrm{CD} 45 \mathrm{RA}+$ ) which show enhanced self-renewal capabilities. ${ }^{39}$ Our results supported, but do not prove, a TRM phenotype in PEB and EST in contrast to the circulating memory (CD45RA+CD69-) peripheral blood phenotype. This was especially the case for CD $8+$ T cells that expressed higher CD103 and CD49a transcript compared with matched CD4+ T cells which emerged from our additional bulk RNAseq analysis. However, recent studies have demonstrated the presence of TRM T cells in different tissues including synovial fluid and gut in SpA patients, ${ }^{4041}$ and therefore whether these cells recirculate between these different sites awaits elucidation. The downregulation of KLF2, an important transcription factor for S1PR1 expression, highlights the potential of entheseal cells in maintaining their tissue retention capabilities and TRM phenotype. Interestingly, the transcription factor Hobit (Homolog of Blimp-1 in T cells, also known as ZNF683), which directs a tissue-resident programme and prevents egress of immune cells, ${ }^{42}$ was downregulated in both CD4+ and CD8 + entheseal $\mathrm{T}$ cells, respectively, compared with peripheral blood. This result contradicts previous findings in mice where Hobit is known to be upregulated, highlighting a difference between human and mouse models and the signature TRM-associated genes. ${ }^{43}$ Unlike other target tissues in SpA such as the gut and skin, where direct interfacing with potential microbial pathogens occurs, the basis for such populations in the normal enthesis in health is poorly understood and may be related to tissue repair and integrity.

In conclusion, this work shows that the normal human enthesis harbours conventional $\mathrm{T}$ cells with inducible production of IL-17 and TNF. The CD $8+$ Tcells at the enthesis displayed markers suggestive of tissue residency, but further single cell RNAseq work is planned to better define this. The results also support the idea that IL-17 production may be independent of IL-23, where the in vitro enthesitis model allows for TCR engagement via CD3/CD28 crosslinking. These findings provide a base for future studies to define the role of adaptive immune cells in the pathogenesis of enthesitis in active early SpA. Further studies are needed to extend these findings into diseased tissue in SpA.

\section{Author affiliations}

${ }^{1}$ University of Leeds Leeds Institute of Rheumatic and Musculoskeletal Medicine, Leeds, UK 
${ }^{2}$ Internal Medicine "B", Sheba Medical Center, Tel Hashomer, Israel

${ }^{3}$ The Zabludowicz Center for Autoimmune Diseases, Rheumatology Unit, Sheba Medical Center, Tel-Hashomer, Israel

${ }^{4}$ Sackler Faculty of Medicine, Tel Aviv University, Tel Aviv, Israel

${ }^{5}$ Department of Rheumatology \& Immunology, Sichuan Academy of Medical Sciences \& Sichuan Provincial People's Hospital, Chengdu, Sichuan, China

${ }^{6}$ Queensland University of Technology, Institute for Health and Biomedical Innovation, Translational Research Institute, Princess Alexandra Hospital, Brisbane, Queensland, Australia

${ }^{7}$ Leeds Teaching Hospitals NHS Trust, Leeds, UK

${ }^{8}$ Universita degli Studi di Genova Dipartimento di Medicina Interna e Specialita Mediche, Genova, Liguria, Italy

${ }^{9}$ Department of Mathematics and Statistics, Laboratory for Industrial and Applied Mathematics (LIAM), York University, Toronto, Ontario, Canada

${ }^{10}$ Leeds Teaching Hospitals NHS Trust, Leeds, UK

${ }^{11}$ Guy's and St Thomas' NHS Foundation Trust, King's College London NIHR Biomedical Research Centre, London, United Kingdom

${ }^{12}$ Division of Haematology and Immunology, University of Leeds, Leeds, UK

Correction notice This article has been corrected since it published Online First. The ORCD ID for Abdulla Watad has been corrected.

Twitter Tony Kenna @tonykenna3

Acknowledgements Thanks to Mark Coles for provision of immunofluorescence microscopy support.

Contributors $A W, C B, R C, D N$ and $D G M$ have contributed to the study planning and design. AW, HR, RC, CB, LA, MW, TK, DN, MB and DGM contributed to the acquisition, analysis or interpretation of data and critical revision of the manuscript. $T R$, QZ, AK, RD, PL, VB, AR, PM, NB, HA, MW, KS, TK, MB, DN and DGM contributed to the acquisition of data and drafting of the manuscript. All the authors have read and approved the final version of the manuscript.

Funding DGM is funded by the Leeds NIHR Biomedical Research Centre. Funding: Novartis UK -investigator-initiated non-clinical research funding support (HR, TR, CB). Research funded by a Pfizer investigator-initiated research grant (RC). AW QZ, and KS were funded by the Celgene supported PARTNER fellowship programme.

Competing interests None declared.

Patient and public involvement Patients and/or the public were not involved in the design, or conduct, or reporting, or dissemination plans of this research.

Patient consent for publication Not required.

Ethics approval The current study has been approved by the University of Leeds ethical approval committee.

Provenance and peer review Not commissioned; externally peer reviewed.

Data availability statement All data relevant to the study are included in the article or uploaded as supplementary information. All the relevant data is included in the article or supplementary file.

Open access This is an open access article distributed in accordance with the Creative Commons Attribution Non Commercial (CC BY-NC 4.0) license, which permits others to distribute, remix, adapt, build upon this work non-commercially, and license their derivative works on different terms, provided the original work is properly cited, appropriate credit is given, any changes made indicated, and the use is non-commercial. See: http://creativecommons.org/licenses/by-nc/4.0/.

\section{ORCID iDs}

Abdulla Watad http://orcid.org/0000-0002-1404-8027

Hannah Rowe http://orcid.org/0000-0002-7135-3255

Matthew A Brown http://orcid.org/0000-0003-0538-8211

Darren Newton http://orcid.org/0000-0002-0214-1486

\section{REFERENCES}

1 Benjamin M, McGonagle D. Entheses: tendon and ligament attachment sites. Scand J Med Sci Sports 2009;19:520-7.

2 Bridgewood C, Watad A, Cuthbert RJ, et al. Spondyloarthritis: new insights into clinical aspects, translational immunology and therapeutics. Curr Opin Rheumatol 2018;30:526-32.

3 Watad A, Bridgewood C, Russell T, et al. The early phases of ankylosing spondylitis: emerging insights from clinical and basic science. Front Immunol 2018;9:2668.

4 Watad A, Cuthbert RJ, Amital H, et al. Enthesitis: much more than focal insertion point inflammation. Curr Rheumatol Rep 2018;20:41.

5 Gracey E, Burssens A, Cambré I, et al. Tendon and ligament mechanical loading in the pathogenesis of inflammatory arthritis. Nat Rev Rheumatol 2020:1-15.

6 Rizzo A, Guggino G, Ferrante A, et al. Role of subclinical gut inflammation in the pathogenesis of spondyloarthritis. Front Med 2018:5:63.

7 Mensah KA, Schwarz EM, Ritchlin CT. Altered bone remodeling in psoriatic arthritis. Curr Rheumatol Rep 2008;10:311-7
8 Jacques $\mathrm{P}$, Lambrecht $\mathrm{S}$, Verheugen $\mathrm{E}$, et al. Proof of concept: enthesitis and new bone formation in spondyloarthritis are driven by mechanical strain and stromal cells. Ann Rheum Dis 2014;73:437-45

9 Braem K, Carter S, Lories RJ. Spontaneous arthritis and ankylosis in male DBA/1 mice: further evidence for a role of behavioral factors in "stress-induced arthritis". Biol Proced 2012:14:10.

10 Sherlock JP, Joyce-Shaikh B, Turner SP, et al. II-23 induces spondyloarthropathy by acting on ROR- $\gamma \mathrm{t}+\mathrm{CD} 3+\mathrm{CD} 4-\mathrm{CD} 8$ - entheseal resident T cells. Nat Med 2012;18:1069-76.

11 Reinhardt A, Yevsa T, Worbs T, et al. Interleukin-23-Dependent $\gamma / \delta$ T cells produce interleukin-17 and accumulate in the Enthesis, aortic valve, and ciliary body in mice. Arthritis Rheumatol 2016;68:2476-86.

12 Cuthbert RJ, Fragkakis EM, Dunsmuir R, et al. Brief report: group 3 innate lymphoid cells in human Enthesis. Arthritis Rheumato/ 2017;69:1816-22.

13 Bridgewood C, Watad A, Russell T, et al. Identification of myeloid cells in the human enthesis as the main source of local IL-23 production. Ann Rheum Dis 2019;78:929-33.

14 Wellcome Trust Case Control Consortium, Australo-Anglo-American Spondylitis Consortium (TASC), Burton PR, et al. Association scan of 14,500 nonsynonymous SNPs in four diseases identifies autoimmunity variants. Nat Genet 2007;39:1329-37.

15 Evans DM, Spencer CCA, Pointon JJ, et al. Interaction between ERAP1 and HLA-B27 in ankylosing spondylitis implicates peptide handling in the mechanism for HLA-B27 in disease susceptibility. Nat Genet 2011;43:761-7.

16 Chatzikyriakidou A, Voulgari PV, Drosos AA. What is the role of HLA-B27 in spondyloarthropathies? Autoimmun Rev 2011;10:464-8.

17 Appel H, Sieper J. Spondyloarthritis at the crossroads of imaging, pathology, and structural damage in the era of biologics. Curr Rheumatol Rep 2008;10:356-63.

18 Schenkel JM, Masopust D. Tissue-Resident memory T cells. Immunity 2014;41:886-97.

19 Gebhardt T, Palendira U, Tscharke DC, et al. Tissue-Resident memory T cells in tissue homeostasis, persistent infection, and cancer surveillance. Immunol Rev 2018;283:54-76.

20 van der Heijde D, Ramiro S, Landewé R, et al. 2016 update of the ASAS-EULAR management recommendations for axial spondyloarthritis. Ann Rheum Dis 2017:76:978-91.

21 Baeten D, Sieper J, Braun J, et al. Secukinumab, an interleukin-17A inhibitor, in ankylosing spondylitis. N Engl J Med 2015;373:2534-48.

22 Baeten D, Østergaard M, Wei JC-C, et al. Risankizumab, an IL-23 inhibitor, for ankylosing spondylitis: results of a randomised, double-blind, placebo-controlled, proof-of-concept, dose-finding phase 2 study. Ann Rheum Dis 2018:77:1295-302.

23 Siebert S, Millar NL, McInnes IB. Why did IL-23p19 inhibition fail in as: a tale of tissues, trials or translation? Ann Rheum Dis 2019:78:1015-8.

24 Bridgewood C, Russell T, Weedon H, et al. The novel cytokine Metrnl/IL-41 is elevated in psoriatic arthritis synovium and inducible from both entheseal and synovial fibroblasts. Clin Immunol 2019:208:108253.

25 Smith SH, Peredo CE, Takeda Y, et al. Development of a topical treatment for psoriasis targeting ROR $\gamma$. from bench to skin. PLoS One 2016;11.

26 Bankovich AJ, Shiow LR, Cyster JG. Cd69 suppresses sphingosine 1-phosophate receptor-1 (S1P1) function through interaction with membrane helix 4. J Biol Chem 2010;285:22328-37

27 Xue X, Soroosh P, De Leon-Tabaldo A, et al. Pharmacologic modulation of ROR $\gamma$ translates to efficacy in preclinical and translational models of psoriasis and inflammatory arthritis. Sci Rep 2016:6:37977.

28 Lubrano E, Massimo Perrotta F, Manara M, et al. Predictors of loss of remission and disease flares in patients with axial spondyloarthritis receiving antitumor necrosis factor treatment: a retrospective study. J Rheumatol 2016:43:1541-6.

29 Infante-Duarte C, Horton HF, Byrne MC, et al. Microbial lipopeptides induce the production of IL-17 in Th cells. J Immuno/ 2000;165:6107-15.

30 Steel KJA, Srenathan U, Ridley M, et al. Polyfunctional, proinflammatory, TissueResident memory phenotype and function of synovial Interleukin-17A+CD 8+ T cells in psoriatic arthritis. Arthritis2020;72:435-47.

31 Flores-Santibáñez F, Cuadra B, Fernández D, et al. In Vitro-Generated Tc17 Cells Present a Memory Phenotype and Serve As a Reservoir of Tc1 Cells In Vivo. Front Immunol 2018:9:209.

32 Schett G, Lories RJ, D'Agostino M-A, et al. Enthesitis: from pathophysiology to treatment. Nat Rev Rheumatol 2017:13:731-41.

33 Benjamin M, McGonagle D. The anatomical basis for disease localisation in seronegative spondyloarthropathy at entheses and related sites. J Anat 2001;199:503-26.

34 Zhong C, Zhu J. Small-Molecule RORyt antagonists: one stone kills two birds. Trends Immunol 2017:38:229-31.

35 Solt LA, Kumar N, Nuhant P, et al. Suppression of Th17 differentiation and autoimmunity by a synthetic ROR ligand. Nature 2011:472:491-4.

36 McGeehan G, Bukhtiyarov Y, Zhao Y, et al. VTP-43742 is a potent and selective ROR $\gamma$ t blocker that demonstrates oral efficacy in a mouse model of autoimmunity through suppression of IL-17A production (THER7P.945). J Immunol 2015;194:205-8.

37 de Wit J, Al-Mossawi MH, Hühn MH, et al. RORyt inhibitors suppress T(H)17 responses in inflammatory arthritis and inflammatory bowel disease. J Allergy Clin Immunol 2016;137:960-3 
38 Venken K, Jacques P, Mortier C, et al. Roryt inhibition selectively targets IL-17 producing iNKT and $\gamma \delta$-T cells enriched in spondyloarthritis patients. Nat Commun 2019;10:9.

39 Gattinoni L, Speiser DE, Lichterfeld M, et al. T memory stem cells in health and disease. Nat Med 2017;23:18-27.

40 Qaiyum Z, Gracey E, Yao Y, et al. Integrin and transcriptomic profiles identify a distinctive synovial CD8+ T cell subpopulation in spondyloarthritis. Ann Rheum Dis 2019;78:1566-75.
41 Guggino G, Rizzo A, Mauro D, et al. Gut-derived CD8 ${ }^{+}$tissue-resident memory T cells are expanded in the peripheral blood and synovia of SpA patients. Ann Rheum Dis 2019:doi: 10.1136/annrheumdis-2019-216456. [Epub ahead of print: 18 Oct 2019].

42 Mackay LK, Minnich M, Kragten NAM, et al. Hobit and Blimp1 instruct a universal transcriptional program of tissue residency in lymphocytes. Science 2016;352:459-63.

43 Kumar BV, Ma W, Miron M, et al. Human tissue-resident memory T cells are defined by core transcriptional and functional signatures in lymphoid and mucosal sites. Cell Rep 2017:20:2921-34 